\title{
BMJ Open Assessment of global coagulation function under treatment with emicizumab concomitantly with bypassing agents in haemophilia A with inhibitor (UNEBI Study): multicentre open-label non-randomised clinical trial
}

\author{
Kenichi Ogiwara (D) , ${ }^{1}$ Masashi Taki, ${ }^{2}$ Takashi Suzuki, ${ }^{3}$ Hideyuki Takedani, ${ }^{4}$ \\ Tadashi Matsushita, ${ }^{5}$ Kagehiro Amano, ${ }^{6}$ Masanori Matsumoto, ${ }^{7}$ Kenji Nishio, ${ }^{8}$ \\ Midori Shima (D) , ${ }^{1}$ Masato Kasahara, ${ }^{9}$ Keiji Nogami (D ${ }^{1}$
}

To cite: Ogiwara K, Taki M, Suzuki T, et al. Assessment of global coagulation function under treatment with emicizumab concomitantly with bypassing agents in haemophilia A with inhibitor (UNEBI Study): multicentre open-label non-randomised clinical trial. BMJ Open 2022;12:e056922. doi:10.1136/ bmjopen-2021-056922

- Prepublication history and additional supplemental material for this paper are available online. To view these files, please visit the journal online (http://dx.doi.org/10.1136/ bmjopen-2021-056922).

Received 31 August 2021 Accepted 28 January 2022

Check for updates

(C) Author(s) (or their employer(s)) 2022. Re-use permitted under CC BY-NC. No commercial re-use. See rights and permissions. Published by BMJ.

For numbered affiliations see end of article.

Correspondence to Professor Keiji Nogami; roc-noga@naramed-u.ac.jp

\section{ABSTRACT}

Introduction Subcutaneous emicizumab prophylaxis substantially reduces bleeding episodes in patients with haemophilia A (PwHA) and factor VIII inhibitor. However, thrombotic events occurred in some PwHA with inhibitor who had received high cumulative doses of activated prothrombin complex concentrates at their breakthrough bleeds, when they were also given prophylactic emicizumab. After that, although the recommended guidance was proposed for bypassing agents (BPAs) therapy under emicizumab prophylaxis for haemostatic management, detailed investigation(s) is(are) required to elucidate the safe and appropriate dose of BPAs to use concomitantly with emicizumab prophylaxis.

Methods and analysis In the UNEBI Study, 60 PwHA with inhibitor will be enrolled for a maximum duration of 3 years, and samples of 20 events following concomitant use of BPAs with emicizumab will be collected. An 'event' is defined as obtaining blood samples before and after administration of BPA when a breakthrough bleed or a surgical procedure occurs. The coagulation potential in the obtained samples will be measured by global coagulation assays. The primary endpoint is the degree of improvement in the maximum coagulation rate by clot waveform analysis (CWA) before and after administration of fixed-dose BPAs. This parameter obtained from CWA, which is triggered with an optimally diluted mixture of prothrombin time/activated partial thromboplastin time-reagents, is reported to be an excellent marker for assessing the degree of improvement in coagulation potential in emicizumab-treated plasma.

Ethics and dissemination The UNEBI Study was approved by the Japan Certified Review Board of Nara Medical University. The results of the study will be communicated through publication in international scientific journals and presentations at (inter)national conferences.

Trial registration number $\mathrm{jRCTs} 051190119$.
Strengths and limitations of this study

- The UNEBI Study is a multicentre, prospective trial including patients from 13 haemophilia treatment centres in Japan.

- We can evaluate the coagulation potentials on the administration of bypassing agent (BPA) concomitant with emicizumab prophylaxis in patients with haemophilia $A$ with inhibitor by using recommended global coagulation assays.

- The UNEBI Study is a single-armed trial, and the effects of improvement in coagulation potential for patients may be heterogeneous.

- Since the choice of BPA for haemostatic treatment depends on the attending physician, there may be differences among the patient numbers for the three types of BPA.

\section{INTRODUCTION}

Haemophilia A (HA) is a congenital bleeding disorder caused by quantitative or qualitative abnormalities of coagulation factor VIII (FVIII). Replacement therapy is essential for haemostatic treatment of bleeding in patients with HA (PwHA), and the development of regular prophylaxis with FVIII products has dramatically improved health-related quality of life (HRQoL) of these patients. ${ }^{1}$ However, some serious concerns remain regarding the current therapeutic protocols. Frequent intravenous infusions of FVIII products often pose a mental and physical burden, especially for paediatric patients and their parents. ${ }^{2-4}$ Moreover, the development of anti-FVIII antibodies (inhibitors) seriously complicates clinical management. FVIII inhibitors render standard FVIII treatment ineffective, and 
make the appropriate haemostatic treatment difficult to determine for these patients. ${ }^{5-7}$

Emicizumab is a humanised, bispecific antibody against activated FIX (FIXa) and FX, which mimics the cofactor function of activated FVIII (FVIIIa) by spatially relocating FIXa and FX to the appropriate position in the tenase

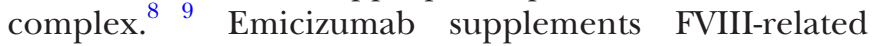
mechanisms regardless of the presence of FVIII inhibitors, is administered subcutaneously and has a long halflife (approximately 30 days). ${ }^{10}$ Therefore, emicizumab has been expected to overcome the limitations of current therapeutic protocols. The effectiveness of emicizumab has been confirmed by several global clinical phase 3 studies of PwHA with or without FVIII inhibitors, ${ }^{11-15}$ resulting in approval for clinical use of emicizumab in the USA, European Union and Japan. As of 2020, more than 6500 PwHA have been introduced to this agent and have gotten a good HRQoL comparable with standard regular prophylaxis.

However, several issues associated with emicizumab prophylaxis have emerged. The most important problem is thrombotic adverse events that occurred when bypassing agents (BPAs) such as activated prothrombin complex concentrates (aPCCs) were administered concomitantly with emicizumab. ${ }^{11}$ In a global phase 3 trial (HAVEN1 ), three cases of thrombotic microangiopathy (TMA) and two cases of thrombotic embolism (TE) occurred. ${ }^{11}$ Since all these patients used aPCC $(>100 \mathrm{U} / \mathrm{kg} /$ day for $>24$ hours) when breakthrough bleeds occurred, it has been recommended that recombinant activated FVII (rFVIIa) should be used as first-line therapy rather than aPCC or other BPAs.

Another difficulty is the limited availability of methods for monitoring the effect of emicizumab. Emicizumab mimics FVIIIa unlike FVIII and does not require activation by thrombin, ${ }^{8}$ resulting in a shortening effect of the antibody, much greater than that of FVIII, on the activated partial thromboplastin time (aPTT) ${ }^{8}{ }^{816}$ In clinical practice, haemostatic monitoring may not be required in stable patients if their bleeds are well controlled. However, haemostatic monitoring should be considered in the presence of sudden increased bleeding rates. In such cases, the change in antibody concentration mediated by gain in body weight or the development of an anti-drug antibody should be ruled out. In addition, comprehensive assessment of clotting function should be undertaken for haemostatic management by concomitant use of BPAs or FVIII concentrates for breakthrough bleeds or surgery.

Haemostatic monitoring of BPAs has been discussed for a long time, and some global assays including thromboelastometry (rotational thromboelastometry (ROTEM)/thromboelastography), ${ }^{17}$ clot waveform analysis $(\mathrm{CWA})^{18}$ and thrombin generation test $(\mathrm{TGT})^{19}$ have been reported to be more appropriate than conventional assays such as aPTT. These assays appear to be useful not only for BPAs but also for emicizumab. ${ }^{20}$ Furthermore, concomitant use of BPAs and emicizumab could also be monitored with these assays. ${ }^{21}$ We have already reported that ROTEM, ${ }^{22} 23 \mathrm{CWA}^{2324}$ and $\mathrm{TGT}^{81025}$ could be used for monitoring the coagulation potential of emicizumab in the presence or absence of BPAs or FVIII concentrates. One of the most important aspects of global assays is a trigger reagent used in these assays. The physiological coagulation reaction is triggered by a minimal amount of tissue factor (TF) ${ }^{26}$ We have developed a combinedtriggered global assay with a minimal amount of TF and ellagic acid to optimise plasma-based global assays (CWA and TGT) for PwHA. 224

A mixture of two anti-emicizumab monoclonal antibodies attached anti-FIX-F $\left(\mathrm{ab}^{\prime}\right)_{2}$ and anti-FX-F $\left(\mathrm{ab}^{\prime}\right)_{2}$ (anti-Emi-mAb) neutralising emicizumab activity has been developed to measure FVIII activity or inhibitor titre in emicizumab-treated patients. ${ }^{27}$ A pilot study suggested that the anti-Emi-mAb could neutralise emicizumab activity even in global assays such as ROTEM, TGT and CWA (unpublished data). These global assays might be used to discriminate emicizumab-derived haemostatic function and BPA-derived haemostatic function in whole blood or plasma of emicizumab-treated patients who have been treated with additional BPAs in breakthrough bleeds or during perioperative management.

Another global haemostatic assay is a flow chamberbased system (T-TAS, Fujimori-Kogyo, Tokyo, Japan) that can evaluate both primary and secondary haemostasis in the presence of blood flow. ${ }^{28} 29$ Because TMA occurs as a result of hyperactivation of primary haemostasis, including the actions of platelets, von Willebrand factor (VWF) and ADAMTS13, ${ }^{30} 31$ this flow-chamber system could be expected to have the potential to evaluate emicizumab-associated TMA/TE events.

To overcome these issues associated with emicizumab treatment mentioned above, we have started a clinical study called 'Assessment of global coagulation function under treatment with emicizumab concomitantly with bypassing agents in haemophilia A with inhibitor' (UNEBI Study), named after a famous historical mountain, Mt Unebi, located in the old Japanese capital (Kashihara, Nara, Japan). This multicentre, prospective trial, including patients from 13 major haemophilia treatment centres in Japan, would contribute to understanding of the comprehensive haemostatic function of emicizumab and BPAs used concomitantly, and might offer a clue as to why TMA/TE develops in a few cases under such conditions.

\section{METHODS AND ANALYSIS}

A summary of the study design is shown in figure 1 . This study started on 6 March 2020 and will end until 30 September 2023.

\section{Aim}

The aim of the UNEBI Study is to evaluate global coagulation function under treatment with emicizumab concomitantly with BPAs in PwHA with inhibitor. Furthermore, the aim is to explore any clues that might contribute to 


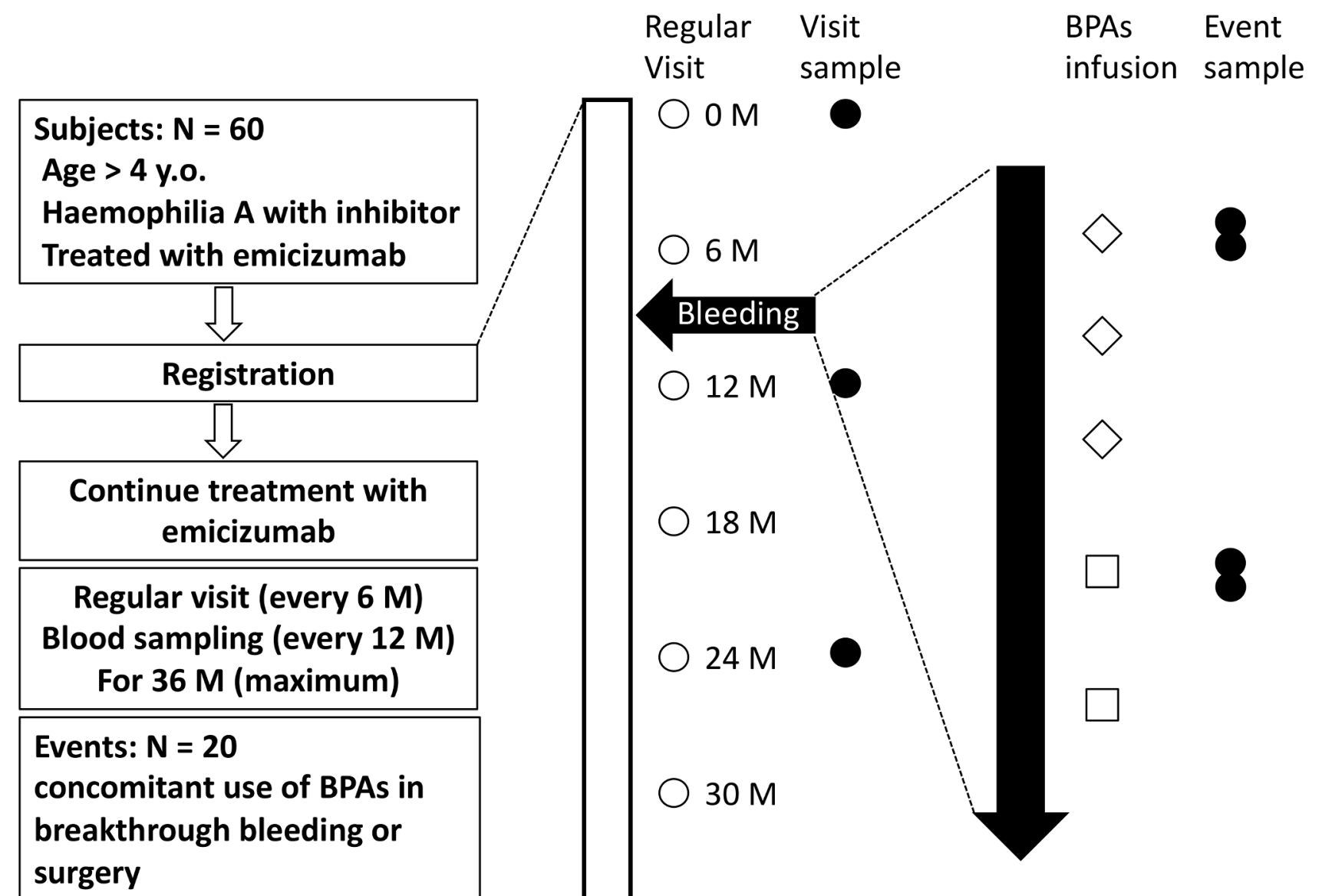

$36 \mathrm{M}$

Figure 1 Schema of the study design. Sixty subjects who are patients with haemophilia A with inhibitor and treated with emicizumab will be registered. Subjects need to visit the participating institutions every 6 months and need to have blood samples withdrawn every 12 months in order to evaluate the single drug effect of emicizumab on global coagulation. If breakthrough bleeds or surgical procedures take place, and the investigator decides that BPA is needed for treatment, blood will be withdrawn at two time points, before and $30 \mathrm{~min}$ after the first infusion of BPA. Usually, rFVIla is the BPA first infused. If other BPAs are considered for use as additional treatment, the first infusion of the switched BPA is also the timing of two-point blood sample collection as an additional event. This study aims to obtain 20 events within 3 years. BPA, bypassing agent; rFVIIa, recombinant activated factor VII concentrates.

our understanding of the mechanism(s) leading to TMA and TE in these settings of concomitant use.

\section{Study subjects}

Sixty of PwHA with inhibitor will be enrolled in this study for a maximum duration of 3 years (from 6 March 2020 to 31 March 2023), and 20 events following concomitant use of BPAs will be collected. An 'event' means that both of blood samples are collected before and after an infusion of BPAs when haemostatic management is performed at breakthrough bleeds or surgery.

\section{Inclusion criteria}

1. Congenital HA with inhibitor over 4 years old.

2. Patients treated with or planned for emicizumab based on the latest package insert.

3. Patients who were informed about the content of this clinical study and provided written consent.

4. Patients who can comply with the planned procedure in this clinical study.
Exclusion criteria

1. Patients who have difficulty in making regular visits and/or visits at the time of an event.

2. Patients with other diseases characterised by abnormal liver function Aspartate aminotransferase (AST) and/ or Alanine aminotransferase (ALT) > fivefold of the upper limit of normal range) or low platelet counts $(<100 \times 109 / \mathrm{L})$.

3. Patients who have difficulty in having blood collected.

4. Patients judged by the investigator to be inappropriate to enter this study for some other reason.

\section{Emicizumab prophylaxis}

Subjects must comply with the last updated recommended use for emicizumab prophylaxis. As of August 2020, the recommended dose is $3 \mathrm{mg} / \mathrm{kg}$ by subcutaneous injection once weekly for the first 4 weeks, followed by one of three options as mentioned below:

- $1.5 \mathrm{mg} / \mathrm{kg}$, once weekly. 
- $3.0 \mathrm{mg} / \mathrm{kg}$, once every 2 weeks.

- $6.0 \mathrm{mg} / \mathrm{kg}$, once every 4 weeks.

\section{Schedule of assessments}

Regular visit and blood sample collection

Subjects need to visit the participating institutions (13 hospitals in Japan) every 6 months to check that their condition is stable and blood samples need to be obtained every 12 months in order to evaluate the single drug effect of emicizumab on global coagulation function under stable conditions.

\section{Therapeutic intervention in breakthrough bleeds}

This study does not have a strict protocol for every concomitant use of BPAs, but when an event occurs, which means collecting blood samples before and after an infusion of BPA, a strict dosing protocol is mandatory.

When the subject visits the institution for treatment of breakthrough bleed, the investigator should check the details how the subject copes with the bleed before the visit and provide appropriate care due to his symptoms and aetiology.

If the investigator decides that a BPA is needed for his treatment, rFVIIa must be chosen and the first dose administered after the hospital visit must be strictly set at $85 \pm 5 \mu \mathrm{g} / \mathrm{kg}$.

When the first infusion of rFVIIa is not sufficient for haemostasis, additional treatment should be considered. Subsequent rFVIIa infusions should comply with a treatment guidance published by a vendor in Japan.

If the investigator considers that other BPAs such as aPCC or a freeze-dried activated human blood coagulation FVII concentrate containing FX (FVIIa/FX*) are indispensable, the first dosing of aPCC must be $45 \pm 5$ units $/ \mathrm{kg}$ and the total amount of aPCC must be less than 100 units $/ \mathrm{kg}$ in a 24-hour period, and the first dosing of FVIIa/FX must be $55 \pm 5 \mu \mathrm{g} / \mathrm{kg}$. *FVIIa/FX is one of BPAs available in Japan which can be used for PwHA with inhibitor including emicizumab-treated patients. No thrombotic events were reported associated with concomitant use of FVIIa/FX with emicizumab as of December 2021.

\section{Therapeutic intervention in perioperative management}

This study does not have a strict protocol for every concomitant use of BPAs, but when an event occurs, which means to collect blood samples before and after an infusion of BPA, a strict dosing protocol is mandatory.

When the subjects are scheduled for an operation and the investigator decides that BPA is needed for perioperative haemostatic management, rFVIIa must be chosen and the first dose must be strictly set at $85 \pm 5 \mu \mathrm{g} / \mathrm{kg}$.

When the first infusion of rFVIIa is not sufficient for perioperative management, additional treatment should be considered. Subsequent rFVIIa infusions should comply with the treatment guidance published by a vendor in Japan.

If the investigator decides that the use of other BPAs such as aPCC or FVIIa/FX is imperative, the first dose of aPCC must be $45 \pm 5$ units $/ \mathrm{kg}$ and the total amount of aPCC must be less than 100 units $/ \mathrm{kg}$ in a 24-hour period, and the first dose of FVIIa/FX must be $55 \pm 5 \mu \mathrm{g} / \mathrm{kg}$.

\section{Blood sample collection in breakthrough bleeds or perioperative} management

Blood samples are taken by the investigator at two time points: (1) before the first infusion of BPAs (usually when venous access route is secured), (2) $30 \pm 15 \mathrm{~min}$ after the first infusion of BPAs (do not use the same venous access route of BPA infusion). Usually, rFVIIa is the BPA first infused. If other BPAs are considered for use in the additional treatment, the first infusion of the switched BPA at a strict dose level should coincide with the two-point blood sample collection as an additional event.

This study targets to obtain 20 events (20 sample pairs of two time-point blood samples before and after BPA infusion) from 60 registered subjects within 3 years.

\section{Sample handling procedure and sample volume}

Blood samples at regular visits (once yearly) or events (see the Blood sample collection section) are preserved as it is for complete blood counts (CBCs) or centrifuged (collect supernatant) for plasma examinations. Both are sent to a laboratory service company (SRL Japan) and immediately tested for CBC and standard plasma tests for coagulation and fibrinolysis (see the Standard laboratory tests section). A part of the plasma samples was stored in a deep freezer at $-80^{\circ} \mathrm{C}$ and transferred to Nara Medical University for global assays (see the Global assays and TMA-associated tests section). The total sample volume in each venepuncture is $15 \mathrm{~mL}$. Additionally, only at Nara Medical University event samples used were $6 \mathrm{~mL}$ greater in order to perform additional global assays using whole blood (see the Whole blood global assays section). Therefore, the total sample amount at an event in Nara Medical University is $21 \mathrm{~mL}$ for each venepuncture.

\section{Schedule-changing criteria, concomitant therapy and after} treatment

Blood sample collection at regular visits should be prolonged for 2 weeks or more from the last day of BPA use.

Drugs listed as contraindications for combined use in package inserts of the protocol drugs are prohibited. FVIII replacement therapy in breakthrough bleeds or perioperative management is not included as an event in this study. No concomitant therapy of coagulation-associated drugs is permitted for use in the 30-minute duration of the event (between two time points of blood collection). Whether the subject should receive additional treatment depends on the investigator. In principle, rFVIIa should be chosen for additional treatment. If several infusions of rFVIIa are ineffective, then switching to aPCC or FVIIa/FX should be considered. The first infusion of the switched BPA can be considered as an event (two timepoint sample collection). When the subject has already performed home infusion treatment with rFVIIa, the first 
infusion of rFVIIa at the hospital can be deemed to be an event.

\section{Observation and test points}

At the beginning of the registry, background information of subjects including age, severity of HA, latest inhibitor level and drug use history of BPAs is recorded in the electronic data capture (EDC) system.

The medical history associated with bleeding is recorded at regular visits every 6 months, and blood samples are obtained every 12 months to obtain blood analysis information in the stable condition.

When the event, breakthrough bleeds or perioperative management occurs, two-point blood samples before/ after the first infusion of BPAs are obtained. Blood examinations (see the Examination lists section) of this study are performed later to evaluate the efficacy and adverse effects of therapy.

Detailed records of the whole treatment course associated with events (breakthrough bleeds or perioperative management) must be obtained, which includes infusion product name, date and dose of all BPAs including those used at home.

Investigators should evaluate the efficacy and adverse events for both protocol treatment (a single infusion of BPAs) and whole treatment course by routine clinical workup, including physical examination and clinical laboratory tests.

\section{Examination lists}

\section{Standard laboratory tests}

White blood cell (WBC), Red blood cell (RBC), haemoglobin, haematocrit, platelets, AST, ALT, lactate dehydrogenase (LDH), total bilirubin, indirect bilirubin, blood urea nitrogen (BUN), creatinine, fibrinogen, plasminogen, fibrinogen digestive products (FDPs), D-dimer, thrombin-antithrombin complex (TAT) and plasminantiplasmin complex (PIC).

\section{Global assays and TMA-associated tests}

TGT, CWA including clot fibrinolysis waveform analysis (CFWA), plasma level of VWF activity (VWF:RCo), antigen (VWF:Ag), VWF multimer analysis and plasma level of ADAMTS13 activity. TGT and CWA/CFWA are performed in the presence or absence of anti-Emi-mAb. VWF multimer analysis is performed only for event samples. Anti-ADAMTS13 antibody is also measured when ADAMTS13 activity is low $(<10 \%){ }^{30}$

\section{Whole blood global assays}

ROTEM, total thrombus formation analysis system (T-TAS) and whole blood platelet aggregometry (Multiplate). These assays are performed with the event samples only at Nara Medical University. ROTEM is performed in the presence or absence of anti-Emi-mAb.

Schedule of the blood examination described in the Examination lists section is summarised in online supplemental table.

\section{Outcome measures}

To date, there has been no standard assay to evaluate BPAs and emicizumab. Recently, ROTEM, CWA and TGT have been reported to be useful for evaluating the global coagulation efficacy of emicizumab. ${ }^{22-26}$ Among them, we expect that CWA is the most versatile assay, since CWA is an easy-to-use method and is able to perform with some commercial, fully automated coagulation machines. ${ }^{18} 24$ Furthermore, by using anti-Emi-mAb, we attempted to visualise the magnitude of how emicizumab and BPAs contribute distinctly to comprehensive coagulation function (figure 2).

\section{Primary endpoint}

Degree of improvement in maximum coagulation rate by CWA before and after administration of fixed-dose BPAs. A parameter of CWA, Imin1l, which is the maximum velocity, triggered by optimally diluted prothrombin time/aPTT reagents, is reported to be an excellent marker to understand how coagulation function is improved in emicizumab-treated plasma (figure 2) ${ }^{24}$

\section{Secondary endpoint}

1. Comparative evaluation with and without the addition of anti-Emi-mAb for changes in comprehensive coagulation parameters before and after administration of BPAs.

2. Efficacy of emicizumab with and without the addition of anti-Emi antibody using comprehensive coagulation tests in regular samples.

3. Clinical evaluation of haemostasis status and occurrence of TMA or TE.

4. Evaluation of general laboratory tests before and after administration of BPAs, and during regular visits

\section{Exploratory endpoint}

Haemostatic evaluation of TMA-related tests (VWF and ADAMTS13).

\section{Safety assessment}

The clinical haemostatic efficacy of concomitant use of BPAs will be evaluated. Additionally, clinical symptoms associated with TMA or TE and laboratory data including platelet count, FDP, D-dimer, PIC and TAT will be checked during the whole treatment course.

\section{Severe adverse event}

Severe adverse event (SAE) will be immediately reported to the corresponding investigator, regardless of the possibility of a causal link to emicizumab or BPAs. SAE will also be announced to Nara Medical University Certified Review Board and Chugai Pharmaceutical Co. A meeting of the Efficacy and Safety Assessment Committee will be held if needed.

\section{Adverse event of special interest}

Adverse event of special interest (AESI) refers to an adverse event that has been strongly associated with emicizumab based on experiences of previous clinical 


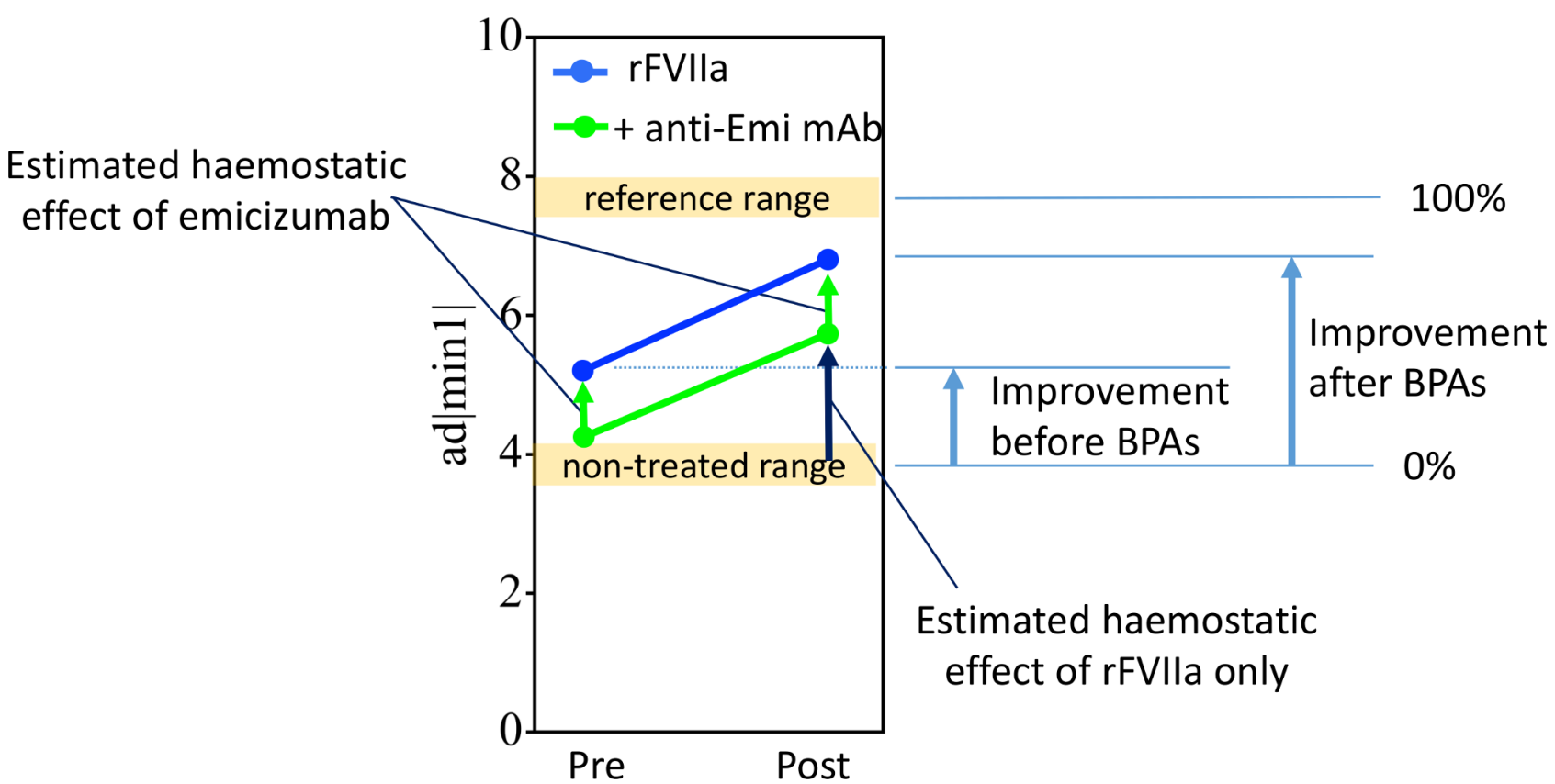

Figure 2 Schema of endpoint parameters. The primary endpoint is the degree of improvement in the maximum coagulation rate by clot waveform analysis before and after administration of BPA (rFVlla in the figure). Furthermore, by using anti-Emi-mAb, we attempted to visualise the relative contribution of emicizumab and BPAs to the magnitude of coagulation function. anti-Emi$\mathrm{mAb}$, anti-emicizumab monoclonal antibodies; BPA, bypassing agent; rFVlla, recombinant activated factor VII concentrates.

trials. The UNEBI Study defines three AESI: (1) systemic hypersensitivity or anaphylactic reaction, (2) TE, and (3) TMA including thrombotic thrombocytopenic purpura or haemolytic uraemic syndrome (HUS). When AESI occurs, the reporting procedure will be the same as for SAE (Severe adverse event section).

\section{Data analysis plan}

\section{Validity of sample size}

Sample size is largely dependent on clinical situation rather than statistical validity. We set the sample size of 60 patients based on the prevalence of $\mathrm{PwHA}$ with inhibitor treated with emicizumab in Japan, number of participating institutions and expected number of acquisitions of written consent. An event number of 20 was determined as the minimum number so that the comparison of parameters shown in primary or secondary endpoints would be statistically valid.

\section{Statistical analysis plan}

For the primary endpoint, the degree of improvement in parameters before and after administration of fixed-dose BPAs will be compared by paired t-test with a significance level of $5 \%$, and estimated after administration level with $95 \%$ CI.

For the secondary endpoint-1, each $95 \%$ CI of with and without the addition of anti-Emi-mAb for changes in comprehensive coagulation parameters before and after administration of BPAs will be estimated. For the secondary endpoint-2, parameters of global assays will be obtained with and without the addition of anti-Emi-mAb in regular samples, and estimated each $95 \%$ CI.
In addition, for the secondary endpoint-3, the haemostasis status and occurrence of TMA or TE will be evaluated and estimated with 95\% CI. For the secondary endpoint -4 , the general laboratory tests before and after administration of BPAs and during regular visits will be evaluated and estimated with $95 \%$ CI.

For the exploratory endpoint, the TMA-related or TE-related parameters (VWF and ADAMTS13) before and after administration of the BPA will be estimated with $95 \%$ CI, and evaluated whether significant change of these parameters will occur.

\section{Patient and public involvement}

Patients and public were not involved in designing this study. Our ethics committee includes public representatives. Results will be presented to patients as part of regular information events.

\section{ETHICS AND DISSEMINATION}

The UNEBI Study was approved by the Japan Certified Review Board of Nara Medical University (29 January 2020) and other participating institutions. The study will be conducted according to the Clinical Trials Act in Japan (Act No. 16 of 14 April 2017) and the Declaration of Helsinki. UNEBI Study corresponds to a criterion of 'Specified clinical trials' based on the Clinical Trials Act since research funds of the study are provided by a manufacturer (Chugai Pharmaceutical Co, Tokyo, Japan) who markets the study drug, emicizumab. The results of this study will be published in peer-reviewed international journals and presented at medical scientific conferences 
in Japanese and/or international societies associated with haemophilia, haematology, or thrombosis and haemostasis. We hope that the results of this study will be adapted in guidelines for haemophilia treatment.

\section{REGISTRATION}

The principal investigator or co-investigator should obtain written informed consent from subjects or their guardians and register them to the EDC system (DATATRAK, ClinCloud, Tokyo, Japan). After confirmation of their eligibility in the EDC system, a registry number is assigned to each subject. Any problems including SAE, withdrawal of consent, research discontinuation and dropout should be immediately reported to the division of the clinical study via the EDC system. The UNEBI Study is registered in the Japan Registry of Clinical Trials (date of registration, 6 March 2020; online site: https://jrct. niph.go.jp/en-latest-detail/jRCTs051190119, last modified on 1 December 2021).

\section{DISCUSSION}

During emicizumab prophylaxis, concomitant therapy using BPAs is required for breakthrough bleeds or surgical procedures. In these settings, although clinicians need to pay attention to possibilities of both undertreatment (insufficient haemostasis) and overtreatment (thrombotic adverse event), a suitable monitoring tool to evaluate haemostatic function comprehensively remains to be established. The UNEBI Study has just started to find the answer to such clinical enigmas.

We have currently reported three methods, ROTEM, CWA and TGT, to monitor the effects of BPAs in emicizumab-treated patients. ${ }^{22-26}$ Another group also reported the usefulness of TGT ${ }^{20}$ and ROTEM $^{20}$ with different trigger conditions. However, few studies have been performed to compare these assays directly. The UNEBI Study will validate the usefulness of these assays with more reliable evidence than previous reports.

In SAEs, the mechanisms leading to TE are easy to understand because the hypercoagulation state would be acceptable during concomitant use of BPAs. However, the mechanism(s) behind the development of TMA remain(s) evasive. TMA is thought to develop due to impaired vascular endothelium, hyperfunction of primary haemostasis associated with higher VWF activity and/ or lower ADAMTS13 activity resulting in an ultra-large VWF multimer and abnormal complement system (atypical HUS) ${ }^{30} 31$ Since the UNEBI Study limits the dosing of BPAs at the lower narrow ranges in only collecting blood samples, TMA and/or TE will be unlikely to occur. However, the study includes TMA-associated tests of VWF and ADAMTS13. Moreover, a flow chamber-based assay that is suitable for detecting abnormal VWF-platelet function ${ }^{28} 29$ will be performed in only one institution. We expect the UNEBI Study to obtain some clues that lead us to ascertain the underlying mechanisms of TMA.
There are several limitations to the UNEBI Study. The sample size is small, although more than half of emicizumab-treated patients with inhibitor in Japan will participate. Blood collection is only once per agent in one bleeding or surgical episode. Global assays using whole blood are limited to one institution, while plasma assays such as CWA and TGT can be performed for all frozen samples. Intervention is very limited because the safety of registered patients is a priority.

\section{Author affiliations}

${ }^{1}$ Pediatrics, Nara Medical University, Kashihara, Japan

${ }^{2}$ Pediatrics, Sei Marianna University School of Medicine Yokohama Seibu Hospital, Yokohama, Japan

${ }^{3}$ Blood Coagulation, Medical Corporation Foundation Ogikubo Hospital, Suginamiku, Japan

${ }^{4}$ Joint Surgery, IMSUT Hospital, Minato-ku, Japan

${ }^{5}$ Blood Transfusion Service, Nagoya University Hospital, Nagoya, Japan

${ }^{6}$ Laboratory Medicine, Tokyo Medical University, Shinjuku-ku, Japan

${ }^{7}$ Blood Transfusion Medicine, Nara Medical University, Kashihara, Japan

${ }^{8}$ General Medicine, Nara Medical University, Kashihara, Japan

${ }^{9}$ Institute for Clinical and Translational Science, Nara Medical University, Kashihara, Japan

\section{Twitter Masashi Taki @Masashi}

Contributors K0, the research secretariat, designed the protocol, prepared figures and wrote the manuscript. MT, the chairman of the protocol committee, designed the protocol. TS, a member of the protocol committee, designed the protocol. HT, a member of the protocol committee, designed the protocol. TM, the chairman of the steering committee, supervised this study. KA, a member of the steering committee, supervised this study. MM, a member of the steering committee, supervised this study. KN, the chairman of the efficacy and safety evaluation committee, supervised this study. MS, a medical advisor, supervised this study. MK, who supports the research secretariat, supervised this study. KN, the principal investigator, designed the protocol, wrote the manuscript and organised this study.

Funding This study was funded by Chugai Pharmaceutical Co (award/grant number: N/A).

Disclaimer This funding source had no role in the design of this study and will not have any role during its execution, analyses, interpretation of the data or decision to submit results.

Competing interests $\mathrm{KO}$ reports grants, personal fees and non-financial support from Chugai Pharmaceutical $\mathrm{C} 0$; grants, personal fees and non-financial support from Sysmex Co, during the conduct of the study; grants from Shire, grants from Takeda Pharmaceutical Co, grants and personal fees from Bioverativ, grants from Sanofi, grants and personal fees from Novo Nordisk, grants and personal fees from Bayer, grants and personal fees from KM Biologics $\mathrm{Co}$, grants and personal fees from CSL Behring Co, outside the submitted work. MT reports grants and personal fees from Chugai Pharmaceutical $\mathrm{Co}$, grants from F Hoffmann-La Roche, during the conduct of the study; personal fees from Takeda Pharmaceutical $\mathrm{Co}$, grants and personal fees from Sanofi, grants and personal fees from Novo Nordisk, personal fees from Bayer, grants and personal fees from CSL Behring $\mathrm{C}$, outside the submitted work. TS reports grants from Chugai Pharmaceutical $\mathrm{Co}$, during the conduct of the study; grants and personal fees from Bayer, grants and personal fees from CSL Behring, grants and personal fees from Novo Nordisk, personal fees from Sanofi, grants and personal fees from Bioverativ, personal fees from Takeda, personal fees from Shire, outside the submitted work. HT reports personal fees from Chugai Pharmaceutical Co, personal fees from Novo Nordisk, personal fees from Sanofi, personal fees from KM Biologics Co, personal fees from Bayer, personal fees from CSL Behring Co, personal fees from Biogen, personal fees from Bioverativ, personal fees from Takeda Pharmaceutical $\mathrm{Co}$, personal fees from Pfizer, outside the submitted work. TM reports personal fees from Baxalta/Shire/Takeda, personal fees from Bayer, grants and personal fees from Novo Nordisk, grants and personal fees from Chugai, grants and personal fees from Pfizer, personal fees from Bioverative/Sanofi, personal fees from CSL, grants and personal fees from JB, grants from KMB, outside the submitted work. KA reports personal fees from Shire, personal fees from Takeda Pharmaceutical Co, personal fees from Bioverativ, personal fees from Sanofi, personal fees from Novo Nordisk, personal 
fees from Bayer, grants and personal fees from KM Biologics Co, personal fees from CSL Behring Co, personal fees from Chugai Pharmaceutical Co, personal fees from Pfizer Japan, personal fees from Japan Blood Products Organization, outside the submitted work. MM reports grants and personal fees from Chugai Pharmaceutical $\mathrm{Co}$, during the conduct of the study; grants and personal fees from Takeda Pharmaceutical Co, grants and personal fees from Sanofi, grants from Bayer, grants from CSL Behring, outside the submitted work. KNishio has nothing to disclose. MS reports grants, personal fees and non-financial support from Chugai Pharmaceutical Co, personal fees from $\mathrm{F}$ Hoffmann-La Roche, personal fees and non-financial support from Sysmex Co, during the conduct of the study; grants from Takeda Pharmaceutical Co, grants from Sanofi, grants from CSL Behring $\mathrm{Co}$, grants from KM Biologics $\mathrm{C}_{0}$, grants from Novo Nordisk, personal fees from Bayer, personal fees from Bioverativ, personal fees from BioMarin Pharmaceutical, outside the submitted work. MK has nothing to disclose. KNogami reports grants, personal fees and non-financial support from Chugai Pharmaceutical Co, grants and personal fees from $\mathrm{F}$ Hoffmann-La Roche, grants, personal fees and non-financial support from Sysmex Co, during the conduct of the study; grants and personal fees from Takeda Pharmaceutical $\mathrm{Co}$, grants and personal fees from Sanofi, grants and personal fees from KM Biologics Co, grants and personal fees from Novo Nordisk, grants and personal fees from Bayer, grants and personal fees from Bioverativ, personal fees from Shire, outside the submitted work.

Patient consent for publication Parental/guardian consent obtained.

Provenance and peer review Not commissioned; externally peer reviewed.

Supplemental material This content has been supplied by the author(s). It has not been vetted by BMJ Publishing Group Limited (BMJ) and may not have been peer-reviewed. Any opinions or recommendations discussed are solely those of the author(s) and are not endorsed by BMJ. BMJ disclaims all liability and responsibility arising from any reliance placed on the content. Where the content includes any translated material, BMJ does not warrant the accuracy and reliability of the translations (including but not limited to local regulations, clinical guidelines, terminology, drug names and drug dosages), and is not responsible for any error and/or omissions arising from translation and adaptation or otherwise.

Open access This is an open access article distributed in accordance with the Creative Commons Attribution Non Commercial (CC BY-NC 4.0) license, which permits others to distribute, remix, adapt, build upon this work non-commercially, and license their derivative works on different terms, provided the original work is properly cited, appropriate credit is given, any changes made indicated, and the use is non-commercial. See: http://creativecommons.org/licenses/by-nc/4.0/.

\section{ORCID iDs}

Kenichi Ogiwara http://orcid.org/0000-0002-3046-6915

Midori Shima http://orcid.org/0000-0002-5922-7061

Keiji Nogami http://orcid.org/0000-0002-2415-2194

\section{REFERENCES}

1 Manco-Johnson MJ, Abshire TC, Shapiro AD, et al. Prophylaxis versus episodic treatment to prevent joint disease in boys with severe hemophilia. N Engl J Med 2007;357:535-44.

2 Beeton K, Neal D, Watson T, et al. Parents of children with haemophilia--a transforming experience. Haemophilia 2007;13:570-9.

3 De Moerloose P, Urbancik W, Van Den Berg HM, et al. A survey of adherence to haemophilia therapy in six European countries: results and recommendations. Haemophilia 2008;14:931-8.

4 du Treil S, Rice J, Leissinger CA. Quantifying adherence to treatment and its relationship to quality of life in a well-characterized haemophilia population. Haemophilia 2007;13:493-501.

5 Fischer K, Lewandowski D, Marijke van den Berg $\mathrm{H}$, et al. Validity of assessing inhibitor development in haemophilia PUPs using registry data: the EUHASS project. Haemophilia 2012;18:e241-6.

6 Giordano P, Lassandro G, Valente M, et al. Current management of the hemophilic child: a demanding interlocutor. Quality of life and adequate cost-efficacy analysis. Pediatr Hematol Oncol 2014;31:687-702.

7 Berntorp E. Differential response to bypassing agents complicates treatment in patients with haemophilia and inhibitors. Haemophilia 2009;15:3-10.
8 Kitazawa T, Igawa T, Sampei Z, et al. A bispecific antibody to factors IXa and $\mathrm{X}$ restores factor VIII hemostatic activity in a hemophilia a model. Nat Med 2012;18:1570-4.

9 Sampei Z, Igawa T, Soeda T, et al. Identification and multidimensional optimization of an asymmetric bispecific lgG antibody mimicking the function of factor VIII cofactor activity. PLoS One 2013;8:e57479.

10 Uchida N, Sambe T, Yoneyama K, et al. A first-in-human phase 1 study of ACE910, a novel factor VIII-mimetic bispecific antibody, in healthy subjects. Blood 2016;127:1633-41.

11 Oldenburg J, Mahlangu JN, Kim B, et al. Emicizumab prophylaxis in hemophilia A with inhibitors. N Engl J Med 2017;377:809-18.

12 Young G, Liesner R, Chang T, et al. A multicenter, open-label phase 3 study of emicizumab prophylaxis in children with hemophilia A with inhibitors. Blood 2019;134:2127-38.

13 Mahlangu J, Oldenburg J, Paz-Priel I, et al. Emicizumab prophylaxis in patients who have hemophilia A without inhibitors. N Engl J Med 2018;379:811-22.

14 Pipe SW, Shima M, Lehle M, et al. Efficacy, safety, and pharmacokinetics of emicizumab prophylaxis given every 4 weeks in people with haemophilia A (HAVEN 4): a multicentre, open-label, non-randomised phase 3 study. Lancet Haematol 2019;6:e295-305.

15 Shima M, Nogami K, Nagami S, et al. A multicentre, open-label study of emicizumab given every 2 or 4 weeks in children with severe haemophilia A without inhibitors. Haemophilia 2019;25:979-87.

16 Shima M, Hanabusa H, Taki M, et al. Factor VIII-mimetic function of humanized bispecific antibody in hemophilia a. $N$ Engl J Med 2016;374:2044-53.

17 Nogami K. The utility of thromboelastography in inherited and acquired bleeding disorders. Br J Haematol 2016;174:503-14.

18 Haku J, Nogami K, Matsumoto T, et al. Optimal monitoring of bypass therapy in hemophilia A patients with inhibitors by the use of clot waveform analysis. J Thromb Haemost 2014;12:355-62.

19 Hoffman M. A cell-based model of coagulation and the role of factor VIla. Blood Rev 2003;17(Suppl 1):S1-5.

20 Adamkewicz JI, Chen DC, Paz-Priel I. Effects and interferences of emicizumab, a humanised bispecific antibody mimicking activated factor VIII cofactor function, on coagulation assays. Thromb Haemost 2019;119:1084-93.

21 Dargaud $\mathrm{Y}$, Lienhart $\mathrm{A}$, Janbain $\mathrm{M}$, et al. Use of thrombin generation assay to personalize treatment of breakthrough bleeds in a patient with hemophilia and inhibitors receiving prophylaxis with emicizumab. Haematologica 2018;103:e181-3.

22 Yada K, Nogami K, Ogiwara K, et al. Global coagulation function assessed by rotational thromboelastometry predicts coagulationsteady state in individual hemophilia $A$ patients receiving emicizumab prophylaxis. Int J Hematol 2019;110:419-30.

23 Furukawa S, Nogami K, Shimonishi N, et al. Prediction of the haemostatic effects of bypassing therapy using comprehensive coagulation assays in emicizumab prophylaxis-treated haemophilia a patients with inhibitors. Br J Haematol 2020;190:727-35.

24 Nogami K, Matsumoto T, Tabuchi Y, et al. Modified clot waveform analysis to measure plasma coagulation potential in the presence of the anti-factor IXa/factor $\mathrm{X}$ bispecific antibody emicizumab. $J$ Thromb Haemost 2018;16:1078-88.

25 Ogiwara K, Nogami K, Matsumoto N, et al. A modified thrombin generation assay to evaluate the plasma coagulation potential in the presence of emicizumab, the bispecific antibody to factors IXa/X. Int $J$ Hematol 2020;112:621-30.

26 Hoffman M, Monroe DM. A cell-based model of hemostasis. Thromb Haemost 2001;85:958-65.

27 Nogami K, Soeda T, Matsumoto T, et al. Routine measurements of factor VIII activity and inhibitor titer in the presence of emicizumab utilizing anti-idiotype monoclonal antibodies. J Thromb Haemost 2018;16:1383-90.

28 Hosokawa K, Ohnishi T, Kondo T, et al. A novel automated microchip flow-chamber system to quantitatively evaluate thrombus formation and antithrombotic agents under blood flow conditions. J Thromb Haemost 2011;9:2029-37.

29 Ogiwara K, Nogami K, Hosokawa K, et al. Comprehensive evaluation of haemostatic function in von Willebrand disease patients using a microchip-based flow chamber system. Haemophilia 2015;21:71-80.

30 Scully M, Cataland S, Coppo P, et al. Consensus on the standardization of terminology in thrombotic thrombocytopenic purpura and related thrombotic microangiopathies. J Thromb Haemost 2017;15:312-22.

31 Wada H, Matsumoto T, Suzuki K, et al. Differences and similarities between disseminated intravascular coagulation and thrombotic microangiopathy. Thromb J 2018;16:14. 